\title{
Digital culture
}

\author{
Alberto Acerbi \\ alberto.acerbi@brunel.ac.uk \\ Centre for Culture and Evolution, Division of Psychology, Brunel University London, Uxbridge, \\ UB8 3PH, United Kingdom
}

For submission to the Oxford Handbook of Cultural Evolution, edited by R. Kendal, J. Tehrani, and J. Kendal (Oxford University Press)

Word count (excluding References): 5815

\begin{abstract}
The diffusion of digital technologies triggered a radical departure from previous modalities of cultural transmission but, at the same time, general characteristics of human cultural evolution and cognition influence these developments. In this chapter, I will explore some areas where the contacts between cultural evolution research and digital media seem more promising. As cultural evolution-inspired research on internet phenomena is still limited, these areas represent suggestions and links with works in other disciplines more than reviews of past research in cultural evolution. These include topics such as how to conceptualise social influence online and how information spreads in social media; how digital media could enhance cumulative culture; and the differences between online and offline cultural transmission. I will then briefly consider possible future directions: the influence of different affordances in different media supporting cultural transmission; the role of producers of cultural traits; and, finally, the effects on cultural dynamics of algorithms selecting information.
\end{abstract}

\section{Keywords}

Cultural evolution; cultural attraction; cultural transmission; digital media; social influence; misinformation; cumulative culture 


\section{Introduction}

Research on the effects of digital media on cultural dynamics is, not surprisingly, abundant and spanning different disciplines. In this chapter, I will focus on research that is inspired by, or compatible with, cultural evolution, intended as a framework encompassing various evolutionary approaches to culture and cognition. Culture evolution is in a privileged position to analyse cultural dynamics in the digital age. Cultural evolution is grounded in a quantitative methodology that promotes the usage of data produced by our digital activities, but it also draws on a robust theoretical background from evolutionary biology and cognitive science, which should help to choose how to use these data and the questions worth asking.

The label "digital culture" is a broad one. Digital media are media encoded in digital format, and their history can be traced back to the first half of the last century, with the invention of digital computers. Here I am discussing a more recent phenomenon, concerning first the diffusion of personal computers, followed by the widespread usage of internet, and finally by the ubiquitous connectivity allowed by portable devices such as smartphones. Below, I will refer interchangeably to "online" or "digital" culture (and media) to address the current stage of these developments.

Despite the rapid growth of the field cultural evolution, research explicitly dedicated to the study of how the diffusion of digital online media impacts culture is still limited within this framework. From one side, general, possibly evolved, features of cognition and common properties of cultural evolution constraint what kind of cultural artefacts succeed and how they develop, in digital media as elsewhere. On the other side, the diffusion of digital and online media impacts cultural dynamics, for example, making it possible to access a virtually unbounded number of individuals from whom to copy from (hyper-availability), or making high-fidelity transmission cheap, quick, and widespread (think to a social media "share"), in a way that was impossible even a few years ago (Acerbi, 2019a, 2016).

In what follows, I will examine some areas where the links between cultural evolution and digital media are starting to be explored, or they seem worth to. More than a review of previous research, the sections below explore ideas to develop, and hopefully suggestions for future works. The ambition is that cultural evolution could provide a promising way to look at these developments, and to 
frame precise research questions. I will first propose that a cultural evolutionary approach, with its emphasis on the adaptivity of social learning and communication, suggests a somewhat reassuring starting point to analyse the effects of social influence online, and I will explore how concepts from cultural evolution can be used to investigate the spread of information in social media. I will then move to one of the central ideas of the framework, that is, cumulative culture, and consider how features of digitally-mediated cultural transmission could impact it. The chapter will then examine how it is possible to compare explicitly cultural transmission offline and online. Finally, I will quickly consider some important topics regarding digital culture, mostly unexplored in cultural evolution: how different online media (e.g. different social media) may support different modalities of transmission; the importance of the intentions of the producers, or spreaders, of cultural traits; and the effects on cultural dynamics of top-down algorithms selecting information for us.

\section{Social influence online}

Research in cultural evolution has extensively explored how we select information to acquire when presented with choices. This stream of research naturally fits with the interest in how information flowing through digital and social media influences us. The hyper-availability provided by online connectivity, coupled with the alleged persuasive power of celebrities, online "influencers," and even algorithmically-based campaigns of mass persuasion, have generated a widespread panic that is often associated with the early phases of the diffusion of new technologies (Orben, 2020). How justified are these concerns?

Social learning is extensively used by humans, and it appears ubiquitous in other species too. One of the central tenets of cultural evolution theory is that for this to be possible, social learning needs to be selective. Drawing on insights from formal modelling showing that indiscriminate social learning is not more effective than individual learning, cultural evolutionists have developed the concept of social learning strategies, or cultural transmission biases, domain-general heuristics used to decide when, what, and from whom to copy (Laland, 2004). Social learning strategies are simple rules, implemented at individual level, such as "copy prestigious people" or "copy the majority." These rules represent a first damper to indiscriminate social influence, offline or online. However, social learning strategies are effective only on average: their efficacy lies explicitly in balancing precision 
and simplicity, so that their outcomes depend on the circumstances.

In this perspective, it could be that the situation created by online social media is so unusual that social learning strategies are not effective in this context (Barkow et al., 2012). This is an interesting question and it will be discussed more in the section Comparing offline and online cultural dynamics, but for now it is important to notice that recent work in cultural evolution is starting to emphasise a more nuanced approach to the usage of social learning strategies. For example, we do not blindly "copy prestigious people," but we copy prestigious people when we can reliably associate their prestige to their success, and in a task we are interested in (Brand et al., 2020). The current view is that social learning strategies are highly flexible, context-dependent, and subject to individual and cultural variations (Kendal et al., 2018). Future work is required to establish whether a consistent theory based on social learning strategies can explain the full variability of empirical observations without losing predictive power.

On top of this, several experiments run by cultural evolutionists have shown that we consistently underuse social information, even when it would be useful for us to copy others (Morin et al., 2021). While the reasons behind this pattern need to be further explored, this strongly goes against the idea of unbounded social influence, both online and offline: the problem seems not to be that we are too gullible, but that we are too stubborn, and we do not accept correct social information enough. Taking these observations into account, the integration of other evolutionary approaches to social influence is a promising direction. Epistemic vigilance has a similar evolutionary rationale, but drawing on communication instead of social learning, considering the possibility of deception and conflicts of interests (scarcely explored in cultural evolution), and drawing on a set of more sophisticated cognitive abilities, such as plausibility checking, trust calibration, and reasoning, used to decide whether to accept information coming from others or not (Mercier, 2020; Sperber et al., 2010).

How can all this be applied to online dynamics? A relevant case study concerns, for example, the spread of misinformation online. While much research has focused, in the past years, on its diffusion and on its purported deleterious effects, when considered within the global information ecosystem the amount of misinformation circulating online is surprisingly limited. Recent empirical studies 
estimated that misinformation represented between around the $0.15 \%$ and the $5 \%$ of the total information circulating on social media (Allen et al., 2020; Grinberg et al., 2019; Guess et al., 2019; Osmundsen et al., 2021). While the exact amount depends on how misinformation is defined (a problem of primary importance by itself), these figures seem consistent with the image of humans as wary or flexible learners delineated by the evolutionary approaches to culture and cognition presented above, more than with one of gullible agents. Even more importantly, engagement with misinformation can not be equated automatically with impact on beliefs and behaviours. Even if in certain contexts misinformation may be shared on social media as much as reliable information (see e.g. de Oliveira \& Albuquerque, 2021), we engage with misinformation to signal political affiliation and group membership, as much as we do with reliable information (Osmundsen et al., 2021), to socialise with our peers (Berriche \& Altay, 2020), simply because of funny or interesting (Acerbi, 2019b) and, in fact, to verify whether it is true, and to say it is not (Tandoc et al., 2018). Various studies show that people engage with political misinformation of their same political slant, so that misinformation can not be considered as a form of influence that changes their beliefs, but as information consistent with their previous ones (Allcott \& Gentzkow, 2017; Guess \& Nyhan, 2018). Regardless of the specific details, a cultural evolutionary approach suggests that we need to be sceptical, at least as a starting point, of gloomy accounts of the dangers of online social influence, and empirical data are mostly consistent with this stance. More importantly, a cultural evolutionary approach provides specific hypotheses that can be tested on online data and specific mechanisms that can be at play when we decide whether to accept or not information, providing a complex picture of social influence, offline and online.

\section{The spread of information online}

Cultural evolution theory makes a broad distinction between two main reasons why a cultural trait spreads. On one side, cultural traits can be successful because of intrinsic characteristics. Everything else being equal, a more effective hammer is likely to spread more than a less effective one. A memorable, attention-grabbing, story is likely to spread more than a dull one. These intrinsic characteristics generate what cultural evolutionists call content-based biases. On the other side, cultural traits can be successful because of contextual reasons, independent from their intrinsic 
features. A not-very-effective hammer can spread because is advertised widely; a dull story can have broad diffusion because a celebrity recounted it. These context-based biases are represented by the heuristics we briefly discussed in the previous section, like "copy the majority," "copy prestigious people," and many others (Kendal et al., 2018).

The traces left by information spreading online can be used to test how cultural traits spread. Most research has focused on content: which features make social media posts or online news successful? This stream of research fits with comparable work in cultural evolution that isolates content that makes narratives successful, often using transmission chain experiments (more on this on Comparing offline and online cultural dynamics). In one of the first works in this area, Heath et al. (2001) found that urban legends containing more elements eliciting disgust (such as the presence of worms in McDonald's hamburgers) were also more diffused in specialised websites. With an explicit cultural evolution background, Acerbi (2019b) analysed the content of online articles classified as misinformation to detect the presence of elements previously highlighted in experiments: disgust, but also threat-related information, negative content, minimally counterintuitive elements, sex-related material, and social information. A main outcome of the analysis was that negative content was preponderant in online misinformation, about five times more common than positive one. The advantage of negative content has been also detected in social media, with an analysis of a large dataset from Twitter, showing that negative tweets were more likely to be retweeted after political events, both negative and positive (Schöne et al., 2021). Similar results were found considering tweets from news organisations: negative affect was expressed more than positive, and predicted more engagement (Bellovary et al., 2021).

The online advantage for negative information is supported by results of transmission chain experiments (Acerbi, 2021; Bebbington et al., 2017) and it is consistent with a broad evolutionary logic for which negative information is more relevant than positive one (Baumeister et al., 2001); however, other studies found that emotional content in general was predictive of online success. For example, analysing New York Times articles, Berger \& Milkman (2012) found that articles with content that generated high-arousal emotions (both negative and positive) were shared more than articles with content evoking low-arousal emotions. Similarly, Brady et al. (2017) observed that the presence of words expressing moral emotions increased the probability for tweets to be retweeted. 
Overall, it could be that both negative content and emotional content in general favour the spread of online information. Further analyses could explicitly investigate how emotional content is linked to the topics that are discussed, such as political or controversial topics, or misinformation versus reliable information. In addition, the intentions of the individual sharing information and the specific context of transmission are likely to be important: sharing New York Times articles with friends, as in Berger \& Milkman (2012) study, may be different than, say, an anonymous conversation on Reddit. Finally, quantitative content analyses of social media spreading have so far focused on emotional content, possibly because it is the easiest to identify automatically with the available software. More sophisticated computational techniques, such as topic modelling or machine learning (as in Brady et al., 2021), allow to detect specific content in large datasets and will enrich our understanding of which content is favoured online.

Less research is dedicated to context-based biases, and practically none within a cultural evolution framework. The role of social media personalities, influencers, and the like, in the spreading of cultural traits online is an open question. A relevant study using Twitter data (Bakshy et al., 2011) found, for example, that the success of a tweet was correlated with the number of followers of the author of the tweet, but this measure can not disentangle the effect of "influence" - similar to demonstrator-based biases in cultural evolutionary theory terms - from pure availability, i.e., the fact that a tweet coming from a user with many followers will simply have more exposition. In addition, the authors noticed that number of followers, together with a second measure called "local influence" (indicating the number of past retweets an user received in the past from their followers), were anyway a poor predictor of future success.

Recent studies have highlighted that successful politically-related content on Twitter is often correlated with out-group animosity or derogation (Osmundsen et al., 2021; Rathje et al., 2021). If this suggests some connection with transmission biases studied in cultural evolution, it is not obvious to square it with the role of strategies like "copying kin" or "copying similar individuals," which are mostly about selecting information.

Similarly, the role of frequency-based biases, such as "copying the majority," is largely unexplored. Popularity in digital online media is often characterised by highly skewed distributions, with very few items that are very popular and many that are not. While this may suggest a reinforcing effect 
of popularity, the mechanisms producing these distributions need to be understood case-by-case, also because such skewed distributions can be produced by unbiased transmission (Bentley et al., 2004).

Finally, a promising methodology to disentangle the role of different transmission biases in online dynamics has recently been proposed by Carrignon et al. (2019). They used individual based models to simulate the expected distributions of retweets given different learning strategies. They then used Approximate Bayesian Computation to compare model outputs with real data: in this way, the transmission bias behind the model that better fit the real data is likely to have generated them in the social media. Analysing a sample of confirmed and debunked rumours (from Vosoughi et al., 2018), Carrignon et al. (2019) concluded that was not possible to find signatures of the transmission biases that they considered, and that an unbiased transmission model had a better fit.

\section{Digital cumulative culture}

Many animals use social cues to adjust their behaviour, but humans are considered the only species to have cumulative culture. While there are various definitions (see e.g. Mesoudi \& Thornton, 2018), the central idea is intuitive: human culture accumulates innovations in a way that is not observed among other species and, iteratively, this process generates cultural traits that would be extremely unlikely to be invented by an isolated individual. Cumulative culture is not an automatic process. Empirical research and models have suggested factors that promote cumulative culture, including large population sizes, efficient social networks, and transmission fidelity (Derex \& Mesoudi, 2020; Lewis \& Laland, 2012).

How does the fact that cultural transmission is now supported by online digital media impact on cumulative culture? A few studies have investigated relevant dynamics in the online domain. Youngblood (2019) tracked the spread of music samples among hip-hop and electronic producers and showed that, thanks to digital sampling technologies, their networks of collaborations are no longer constrained by geographical proximity. Müller \& Winters (2018) analysed the evolution of Reddit Place, a social media experiment in which any registered user could place a single coloured pixel on a 1,000 x 1,000 pixels online digital canvas every 5 to 20 minutes for three days. The 
project involved 1 million individuals that placed more than 16 million pixels. The analysis tracked the increase in compressible graphic patterns, showing that the canvas moved towards a structured state, with many relatively stable and independent patterns.

These studies confirm that digital online media do increase the potential network where cultural transmission can happen and that, at least in some circumstances, online collaboration among many unrelated individuals can be successful. Cultural evolutionists, inspired by population genetics, have developed the concept of effective cultural population size. Effective cultural population size indicates the number of people potentially or actually involved in cultural transmission. The bigger the effective cultural population size, the more likely complex cultural traits are not lost, and effective inventions retained, promoting cumulative cultural evolution (Derex \& Mesoudi, 2020). While the growth of effective cultural population size is a historical process, and it has been impacted by other technological innovations, such as writing, printing, or the increasing accessibility of travel, online media have a tremendous impact, with virtually everyone in western countries having daily internet access. When considering global usage, marked differences remain, but the gap is narrowing, with many countries showing significant growth. Incidentally, what will be the consequences of the diffusion of technologies related to internet to the majority of people on the planet is a compelling question for the next years (Arora, 2019)

Another aspect that links cumulative cultural evolution and the diffusion of digital media is that digital media provide a cheap, fast, and effective way to transmit information. Empirical results suggest that fidelity of transmission is impacted by several factors and that high-fidelity transmission cannot be considered the default condition. Cultural transmission chain experiments, for example, show that oral transmission is generally a low-fi process, with information getting lost during the process (Mesoudi \& Whiten, 2008). As for small effective population sizes, the same risk is present with low transmission fidelity: complex cultural traits can get lost in the process of transmission, negatively impacting cumulative cultural evolution. Transmission fidelity can be, however, increased by "fidelity amplifiers" (Acerbi, 2019a) associated with the process of transmission. Cognitively attractive features can make some narratives easier to remember (J. M. Stubbersfield et al., 2017), rhymes and repetitions can help the memorisation and repetition of stories, written instructions can be preserved better than oral ones. In this perspective, digital media provide several fidelity 
amplifiers: they possess the features that make analogue writing effective, but also many others, including the possibility of direct interactions (e.g. comments associated with an online recipe) or to easily include different media (e.g. YouTube video tutorials).

Increased effective cultural population size and fidelity of transmission do not guarantee improvements in cultural cumulation. It has been proposed that more than size is the typology of the social networks that affects cumulative cultural evolution (Derex \& Mesoudi, 2020). Likewise, fidelity of transmission (coupled with cheap storage) could simply, as it happens, increase exponentially the size of the long tail of cultural traits that are preserved, but not contributing to any process of cultural cumulation. For any successful, and possibly effective in cultural transmission, YouTube video tutorial, there are millions of unwatched videos online.

Few studies have explicitly considered the effect of digital media on cumulative cultural evolution. In a preliminary work, Pianzola et al. (2020) investigated the case of online fan fiction, where non-professional authors expand narratives from existing works of fiction. Fan fiction is an interesting case, because it has been made possible, in its contemporary form, by online media, because is strongly collaborative, and because concerns a domain - art - where the extent of cultural cumulation is debated (Tinits \& Sobchuk, 2020). Pianzola et al. (2020) found that stories in Harry Potter fan fiction accumulated cultural traits (measured as unique tags) through time, suggesting an increase in complexity, and that more recent stories were more liked than earlier ones, suggesting a possible improvement, two features that are often associated to cumulative cultural evolution.

Further research is needed to investigate whether, and in which conditions, digital online media can impact, positively or negatively, cumulative cultural evolution. The availability of data regarding online communities represents by itself an advantage, and these data could be used to test specific hypotheses about, for example, demography and cumulative cultural evolution. To pinpoint the specific role of digital media, it would be important to compare the features of cumulative cultural evolution in similar domains, but with or without digital support. Another possibility is to explicitly design experiments in which skills are transmitted in "traditional" forms of transmission, such as oral descriptions or observations of demonstrators, and in form of transmission made possible by digital online media, such, for example, instructional videos that can be replayed and paused. 


\section{Comparing offline and online cultural dynamics}

In the sections Social influence online and The spread of information online, we mostly discussed how general cultural evolution findings can be applied to the online domain. In the following section, Digital cumulative culture, we started to explore how features of digital cultural transmission, such as the hyper-availability provided by online connectedness and the increased fidelity of transmission, can impact on cultural evolution itself. In this section, we will expand on this and consider more explicitly some differences between offline and online cultural dynamics.

Another feature that characterises online cultural transmission can be called "explicitness." Social media posts are accompanied by information such as the user name of the person that posted it; the number, and often the names, of users that liked or shared (the terminology varies in different social media) the message; comments on the post from other users. Products, including cultural products, such are recipes or songs, are scored, rated, and reviewed publicly. Given the abundance of information available online, we are often presented with the top- $\mathrm{N}$ list of the items we are interested in, as scored by other users or, more likely, by algorithms.

As mentioned above, when evaluating social information, we use cues to infer from where this information is from, or who else is using it. These cues are mostly implicit. One strategy commonly studied in cultural evolution is conformity, technically defined as a disproportionate tendency to copy the majority, meaning that we should have a probability to copy a cultural trait higher than its frequency (to simply "copy the majority," it is sufficient copying at random, since a common behaviour will have more probability to be selected). In a traditional setting, the majority needs to be inferred from a sample of observations: what are other people doing? But in social media we can have an explicit, immediate, and precise quantification: how many people liked or shared a post? What are today's "trending topics?" Something similar happens for "copy prestigious people." In the cultural evolution account, we need to observe to whom other people pay respect or deference to infer prestige, and act accordingly. With online media, we can access effortlessly to information provided by global celebrities or politicians, or we can directly quantify "prestige," for example with the number of followers one user has.

What are the consequences for cultural evolution? One could hypothesise that explicit cues, for 
example of popularity or prestige, could increase our reliance on these strategies, or even make their output more reliable, as their usage is based on accurate assessments. On the other side, the introduction of such explicit cues is a very recent phenomenon, and we may still need to culturally evolve the appropriate tools to make sense of them. A few studies that tried to directly address the question suggest that the effect of explicit cues does not result in a reinforcement of the tendencies to copy the majority or prestigious people. Acerbi \& Tehrani (2018) found, in an online experiment, that participants did not prefer quotes attributed to famous authors (as opposed to random names), and only partially followed popularity cues: they preferred quotes that were presented as chosen by many other participants, but not in the "disproportionate" way that characterises conformity. In an influential paper, Salganik (2006) describe the result of a large-scale experiment, where participants were divided into separated "worlds," all of them listening to the same pool of previously unknown songs. Songs were rated by participants but, here is the catch, the ratings were only shared within worlds. The results show that different songs became successful in different worlds, driven by popularity ratings. At the same time, however, there was a correlation between the success of the songs and their success in a control condition, where ratings were not shown, demonstrating that the information on popularity was only partially driving the dynamics. The effect of explicit cues is an open question, awaiting more research, possibly using also non-experimental data, e.g. from social media, as well as considering cultural traits with tangible effects, unlike listening to songs or choosing preferred quotes.

Another aspect that differentiates online and offline transmission, which we already considered, is fidelity. As we mentioned above, most of the results about what content is favoured in cultural transmission come from transmission chain experiments. Transmission chain experiments are controlled version of the telephone game, where participants need to hear (or read) a story from another participant, memorise it, and repeat it to the next participant in the chain. This is very different to what happens in online transmission, where one does not need to memorise and repeat, but only choose whether to further share, and sometimes willingly modify, something or not.

Some experiments are starting to address the difference in the details of the process of transmission. Eriksson \& Coultas (2014) divided the transmission into three phases, "choose-to-receive" (do participants want to read a story or not?), "encode-and-retrieve" (the standard transmission chain 
procedure) and "choose-to-transmit" (do participants want to transmit the story they read or not?), and found that the content they were interested in (disgust) was favoured in all phases, even when considered separately. J. M. Stubbersfield et al. (2015) and van Leeuwen et al. (2018) also used a similar set-up to test the effect of various types of content in the different phases of transmission. J. Stubbersfield et al. (2018) asked instead participants to purposely modify the content of the material when transmitting it, to make it more appealing. Acerbi (2021) directly compared content effects in a set-up similar to standard transmission chains versus online sharing, and found that, while negative content was favoured in both, results were less conclusive for content elicit disgust and threat-related content. A possible suggestion from this last study is that content biases could be stronger when information needs to be memorised and repeated than when it is not needed. This is consistent with the idea that content biases influence cognitive processing and reproduction but, possibly counterintuitively, suggest that online sharing should be less subject to cognitive content biases than oral transmission.

More broadly, studies that explicitly compare offline and online cultural dynamics are also important to establish causal relationships between the effects we observe and the fact that cultural transmission is digitally supported. Above we highlighted, for example, that negative content is favoured in social media, but this seems to be a more general property of transmission. that we observe also in other contexts. The claim that social media, by themselves, favour negative content is thus probably unwarranted. Similar claims, such as that social media favour the spread of misinformation, emotional content, or similar, need to be evaluated in the same way. Controlled experiment can isolate the features of social media that one considers as important for the effect, be them built-in fidelity (as in the previous example), speed and ease of diffusion, the possibility of feedback ("likes," "share"), and check whether the effect persists or not when they are not included in the transmission set-up. 


\section{Other directions}

\section{Affordances of different media}

In the previous section, we discussed that it is important to take into account the specific features of online transmission. We cannot automatically transfer in the digital realm what we know about cultural transmission in general and, conversely, we cannot conclude from the observation of something happening in the digital realm that this is caused by the digital support. One can also go further, and differentiate among different digital media, or even different social media. At the beginning of this chapter, we pointed up that the "digital culture" label is a broad one. "Digital" cultural transmission can be various social media, emails, online chats, sharing newspapers articles or, indeed, posting an academic chapter on a preprint server or in an online published collection. All these activities imply different interests, digital affordances, and people involved.

Consider, for example, the research mentioned above, showing that the most shared New York Times articles were not characterised by negative emotion, but they are awe-inspiring (Berger \& Milkman, 2012). This goes against the seemingly robust finding of the advantage of negative information in social media. How can we make sense of this discrepancy? One possibility is to consider that we are dealing with two different contexts. Sharing New York Times articles with friends, by email (as considered in this research) is a very different activity than posting something on social media. In the former we are specifically targeting someone, and this someone is known to us, and possibly we expect their reaction, and so on. In the latter, the target is indiscriminate, and we can even be anonymous. In addition, subscribers of the New York Times are a very specific demographic, probably more educated than average, and more left-leaning. Could these differences explain the different outcomes?

This is a single example, but the same holds at various levels, even for different social media. Instagram is optimised for posting, sharing, and editing pictures, TikTok for videos, and Twitter mostly for short texts. Messaging services, such as WhatsApp, are altogether different. While in some occasions it is useful to zoom-out and test general claims about "online" or "digital" culture, in others we may want to be more specific, and consider the fine-grained affordances offered by social/digital media and who their users are. 


\section{The role of producers of cultural traits}

To explain the success of cultural traits, cultural evolutionists generally focus on the interests of the receivers. Social learning strategies are heuristics used by the receivers of cultural traits to decided whether to copy or not. The attractive features of content mentioned in this chapter, such as negative content, threat-related information, and so on, are considered attractive to the receivers. It has recently been suggested that the role of producers of cultural traits has been overlooked, and that it is essential to explain cultural dynamics (André et al., 2020).

Digital media, as much as they changed the way in which information is transmitted (which has been the main focus of this chapter), undoubtedly also changed how information is produced. As cliché as it is, online digital media did allow an unprecedented number of people to record and share information. Social media users are sensitive to the reactions to their activity (Lindström et al., 2021), and we need to take into account their interests when considering online cultural dynamics.

For example, there does not need to be a correlation between what is consumed and what is shared (see e.g. Bright, 2016). The two behaviours are very different but, still, we use hypotheses about what users want to receive to explain cultural success online, which is also, or mostly, determined by what users want to share. Reputational management may have an important role in online dynamics, where information is potentially available to a large number of other people. (Altay et al., 2020) explained the limited spread of online misinformation (see section Social influence online), with the fact that, even if some pieces of information may be attractive for users, sharing them could be detrimental to their reputation, exemplifying a clear contrast between the interests of the same users as consumers or producers (sharers) of cultural traits.

\section{Algorithms}

In this chapter, we discussed how the inclinations and the interests of consumers (and producers) of cultural traits, together with the features of digital media, determine the dynamics of the online spread of information. However, we did not consider what is probably one of the strongest influence of the digital environment, and certainly a radical cultural evolutionary novelty: the algorithmic control of the information we have access to. Algorithms are necessary to select information among the enormous amount that is present in internet or in a specific social media. However, the way 
algorithms do it are bound to have strong consequences on which cultural traits are successful.

One would expect, given that the interest of social media platforms is keeping users "hooked" as much as possible, that the algorithms implemented would amplify the tendencies that we examined above. Thus, to exemplify, if we tend to prefer negative information, an effective (from the platform point of view) algorithm should present, on average, more negative information. However, it is unclear what are the consequences of this amplification, and if it works in practice: as negative information already capture users' attention, presenting too much of it could be futile, or even having the opposite consequences.

In addition, one could legitimately argue that the goal of algorithms implemented by social media should not be hooking users, but something else, and that algorithms should be planned openly and collectively. In this case, the knowledge accumulated in evolutionary approaches to culture could be essential to design and test algorithms that select information online. The main problem for this direction of research is that while some social media (e.g. Twitter) have been open when sharing data about the information circulating on the platform, there is much less openness regarding the algorithms operating.

\section{Acknowledgements}

Some of the ideas discussed here (especially in the section Other directions), were stimulated by the discussion in a "Cultural Evolution in the Digital Age" Book Club organised by the International Cognition \& Culture Institute in 2020. I would like to thank the organisers, Tiffany Morisseau and Dan Sperber, and all the participants. 


\section{References}

Acerbi, A. (2021). From storytelling to facebook. Content biases when retelling or sharing a story. http://dx.doi.org/10.31219/osf.io/br56y

Acerbi, A. (2019a). Cultural evolution in the digital age. Oxford University Press.

Acerbi, A. (2016). A cultural evolution approach to digital media. Frontiers in Human Neuroscience, 10,636 .

Acerbi, A. (2019b). Cognitive attraction and online misinformation. Palgrave Communications, 5, 15. https://doi.org/10.1057/s41599-019-0224-y

Acerbi, A., \& Tehrani, J. J. (2018). Did einstein really say that? Testing content versus context in the cultural selection of quotations. Journal of Cognition and Culture, 18(3-4), 293311.

Allcott, H., \& Gentzkow, M. (2017). Social Media and Fake News in the 2016 Election. Journal of Economic Perspectives, 31(2), 211-236. https://doi.org/10.1257/jep.31.2.211

Allen, J., Howland, B., Mobius, M., Rothschild, D., \& Watts, D. J. (2020). Evaluating the fake news problem at the scale of the information ecosystem. Science Advances, 6(14), eaay3539. https://doi.org/10.1126/sciadv.aay3539

Altay, S., Hacquin, A.-S., \& Mercier, H. (2020). Why do so few people share fake news? It hurts their reputation. New Media \&S Society, 146144482096989. https://doi.org/10.1177/1461444820969893

André, J.-B., Baumard, N., \& Boyer, P. (2020). The mystery of symbolic culture: What fitness costs? What fitness benefits? https://doi.org/10.31219/osf.io/kdh7t

Arora, P. (2019). The Next Billion Users: Digital Life Beyond the West. Harvard University Press.

Bakshy, E., Hofman, J. M., Mason, W. A., \& Watts, D. J. (2011). The fourth ACM international conference. https://doi.org/10.1145/1935826.1935845

Barkow, J. H., O'Gorman, R., \& Rendell, L. (2012). Are the new mass media subverting cultural transmission? Review of General Psychology, 16 (2), 121-133. https://doi.org/10.1037/a0027907

Baumeister, R. F., Bratslavsky, E., Finkenauer, C., \& Vohs, K. D. (2001). Bad is Stronger than Good. Review of General Psychology, 5(4), 323-370. https://doi.org/10.1037/1089-2680.5.4.323

Bebbington, K., MacLeod, C., Ellison, T. M., \& Fay, N. (2017). The sky is falling: evidence of a negativity bias in the social transmission of information. Evolution and Human Behavior, 38(1), 92-101. https://doi.org/10.1016/j.evolhumbehav.2016.07.004

Bellovary, A. K., Young, N. A., \& Goldenberg, A. (2021). Left- and Right-Leaning News Organizations Use Negative Emotional Content and Elicit User Engagement Similarly. Affective Science. https://doi.org/10.1007/s42761-021-00046-w

Bentley, R. A., Hahn, M. W., \& Shennan, S. J. (2004). Random drift and culture change. Proceedings of the Royal Society of London. Series B: Biological Sciences, 271(1547), 14431450. https: //doi.org/10.1098/rspb.2004.2746

Berger, J., \& Milkman, K. L. (2012). What Makes Online Content Viral? Journal of Marketing Research, 49(2), 192-205. https://doi.org/10.1509/jmr.10.0353 
Berriche, M., \& Altay, S. (2020). Internet users engage more with phatic posts than with health misinformation on Facebook. Palgrave Communications, 6(1), 1-9. https://doi.org/10.1057/s4 1599-020-0452-1

Brady, W. J., McLoughlin, K., Doan, T. N., \& Crockett, M. J. (2021). How social learning amplifies moral outrage expression in online social networks. Science Advances, 7(33), eabe5641. https://doi.org/10.1126/sciadv.abe5641

Brady, W. J., Wills, J. A., Jost, J. T., Tucker, J. A., \& Van Bavel, J. J. (2017). Emotion shapes the diffusion of moralized content in social networks. Proceedings of the National Academy of Sciences, $114(28), 7313-7318$.

Brand, C. O., Heap, S., Morgan, T. J. H., \& Mesoudi, A. (2020). The emergence and adaptive use of prestige in an online social learning task. Scientific Reports, 10(1), 12095. https: //doi.org/10.1038/s41598-020-68982-4

Bright, J. (2016). The Social News Gap: How News Reading and News Sharing Diverge. Journal of Communication, 66(3), 343-365. https://doi.org/10.1111/jcom.12232

Carrignon, S., Bentley, R. A., \& Ruck, D. (2019). Modelling rapid online cultural transmission: evaluating neutral models on Twitter data with approximate Bayesian computation. Palgrave Communications, 5(1), 1-9. https://doi.org/10.1057/s41599-019-0295-9

de Oliveira, D. V. B., \& Albuquerque, U. P. (2021). Cultural Evolution and Digital Media: Diffusion of Fake News About COVID-19 on Twitter. SN Computer Science, 2(6). https: //doi.org/10.1007/s42979-021-00836-w

Derex, M., \& Mesoudi, A. (2020). Cumulative Cultural Evolution within Evolving Population Structures. Trends in Cognitive Sciences, 24(8), 654-667. https://doi.org/10.1016/j.tics.2020.04 .005

Eriksson, K., \& Coultas, J. C. (2014). Corpses, Maggots, Poodles and Rats: Emotional Selection Operating in Three Phases of Cultural Transmission of Urban Legends. Journal of Cognition and Culture, 14(1-2), 1-26. https://doi.org/10.1163/15685373-12342107

Grinberg, N., Joseph, K., Friedland, L., Swire-Thompson, B., \& Lazer, D. (2019). Fake news on Twitter during the 2016 U.S. presidential election. Science, 363(6425), 374-378. https: //doi.org/10.1126/science.aau2706

Guess, A., Nagler, J., \& Tucker, J. (2019). Less than you think: Prevalence and predictors of fake news dissemination on Facebook. Science Advances, 5(1), eaau4586. https://doi.org/10.1126/sc iadv.aau 4586

Guess, A., \& Nyhan, B. (2018). Selective exposure to misinformation: Evidence from the consumption of fake news during the 2016 u.s. Presidential campaign. European Reseach Council, 9(3), 4.

Heath, C., Bell, C., \& Sternberg, E. (2001). Emotional selection in memes: The case of urban legends. Journal of Personality and Social Psychology, 81(6), 1028-1041. https://doi.org/10.1037/00223514.81.6.1028

Kendal, R. L., Boogert, N. J., Rendell, L., Laland, K. N., Webster, M., \& Jones, P. L. (2018). Social learning strategies: Bridge-building between fields. Trends in Cognitive Sciences, 22(7), 651665.

Laland, K. N. (2004). Social learning strategies. Learning and Behavior, 32, 4-14. 
Lewis, H. M., \& Laland, K. N. (2012). Transmission fidelity is the key to the build-up of cumulative culture. Philosophical Transactions of the Royal Society B: Biological Sciences, 367(1599), 2171-2180. https://doi.org/10.1098/rstb.2012.0119

Lindström, B., Bellander, M., Schultner, D. T., Chang, A., Tobler, P. N., \& Amodio, D. M. (2021). A computational reward learning account of social media engagement. Nature Communications, 12(1). https://doi.org/10.1038/s41467-020-19607-x

Mercier, H. (2020). Not Born Yesterday: The Science of Who We Trust and What We Believe. Princeton University Press.

Mesoudi, A., \& Thornton, A. (2018). What is cumulative cultural evolution? Proceedings of the Royal Society B: Biological Sciences, 285(1880), 20180712. https://doi.org/10.1098/rspb.2018.0712

Mesoudi, A., \& Whiten, A. (2008). The multiple roles of cultural transmission experiments in understanding human cultural evolution. Philosophical Transactions of the Royal Society B: Biological Sciences, 363(1509), 3489-3501. https://doi.org/10.1098/rstb.2008.0129

Morin, O., Jacquet, P. O., Vaesen, K., \& Acerbi, A. (2021). Social information use and social information waste. Philosophical Transactions of the Royal Society B: Biological Sciences, 376 (1828), 20200052. https://doi.org/10.1098/rstb.2020.0052

Müller, T. F., \& Winters, J. (2018). Compression in cultural evolution: Homogeneity and structure in the emergence and evolution of a large-scale online collaborative art project. PLOS ONE, 13(9), e0202019. https://doi.org/10.1371/journal.pone.0202019

Orben, A. (2020). The Sisyphean Cycle of Technology Panics. Perspectives on Psychological Science, 15(5), 1143-1157. https://doi.org/10.1177/1745691620919372

Osmundsen, M., Bor, A., Vahlstrup, P. B., Bechmann, A., \& Petersen, M. B. (2021). Partisan Polarization Is the Primary Psychological Motivation behind Political Fake News Sharing on Twitter. American Political Science Review, 1-17. https://doi.org/10.1017/s0003055421000290

Pianzola, F., Acerbi, A., \& Rebora, S. (2020). Cultural accumulation and improvement in online fan fiction. 2-11. https://doi.org/10.31219/osf.io/4wjnm

Rathje, S., Van Bavel, J. J., \& van der Linden, S. (2021). Out-group animosity drives engagement on social media. Proceedings of the National Academy of Sciences, 118(26), e2024292118. https://doi.org/10.1073/pnas.2024292118

Salganik, M. J. (2006). Experimental Study of Inequality and Unpredictability in an Artificial Cultural Market. Science, 311 (5762), 854-856. https://doi.org/10.1126/science.1121066

Schöne, J., Parkinson, B., \& Goldenberg, A. (2021). Negativity spreads more than positivity on twitter after both positive and negative political situations. https://doi.org/10.31234/osf.io/x9e7u

Sperber, D., Clément, F., Heintz, C., Mascaro, O., Mercier, H., Origgi, G., \& Wilson, D. (2010). Epistemic vigilance. Mind ES Language, 25(4), 359-393. https://doi.org/10.1111/j.14680017.2010.01394.x

Stubbersfield, J. M., Flynn, E. G., \& Tehrani, J. J. (2017). Cognitive evolution and the transmission of popular narratives: A literature review and application to urban legends. Evolutionary Studies in Imaginative Culture, 1(1), 121. https://doi.org/10.26613/esic.1.1.20 
Stubbersfield, J. M., Tehrani, J. J., \& Flynn, E. G. (2015). Serial killers, spiders and cybersex: Social and survival information bias in the transmission of urban legends. British Journal of Psychology, 106(2), 288-307. https://doi.org/10.1111/bjop.12073

Stubbersfield, J., Tehrani, J., \& Flynn, E. (2018). Faking the news: Intentional guided variation reflects cognitive biases in transmission chains without recall. Cultural Science Journal, 10(1), 54. https://doi.org/10.5334/csci.109

Tandoc, E. C., Lim, Z. W., \& Ling, R. (2018). Defining "Fake News": A typology of scholarly definitions. Digital Journalism, 6(2), 137-153. https://doi.org/10.1080/21670811.2017.1360143

Tinits, P., \& Sobchuk, O. (2020). Open-ended cumulative cultural evolution of Hollywood film crews. Evolutionary Human Sciences, 2. https://doi.org/10.1017/ehs.2020.21

van Leeuwen, F., Parren, N., Miton, H., \& Boyer, P. (2018). Individual choose-to-transmit decisions reveal little preference for transmitting negative or high-arousal content. Journal of Cognition and Culture, 18(1-2), 124-153. https://doi.org/10.1163/15685373-12340018

Vosoughi, S., Roy, D., \& Aral, S. (2018). The spread of true and false news online. Science, 359 (6380), 1146-1151. https://doi.org/10.1126/science.aap9559

Youngblood, M. (2019). Cultural transmission modes of music sampling traditions remain stable despite delocalization in the digital age. PLOS ONE, 14 (2), e0211860. https://doi.org/10.1371/ journal.pone.0211860 